\title{
Resonantly damped oscillations of two coronal loops
}

\author{
D. Robertson and M. S. Ruderman
}

\begin{abstract}
School of Mathematics and Statistics, University of Sheffield, Hicks Building, Hounsfied Road, Sheffield, S3 7RH, UK e-mail: M.S.ruderman@sheffield.ac.uk
\end{abstract}

Received 4 August 2010 / Accepted 27 September 2010

\section{ABSTRACT}

\begin{abstract}
Transverse oscillations of coronal magnetic loops are routinely observed during the space missions. Since the first observation these oscillations were interpreted in terms of kink oscillations of magnetic tubes. Sometimes collective oscillations of two or more coronal loops are observed. This makes the development of theory of collective oscillations of a few parallel magnetic tubes desirable. Another reason for the development of this theory is that there are evidences that at least some coronal loops are not monolithic but consist of many thin magnetic threads. In this paper the linear theory of resonant damping of kink oscillations of two parallel magnetic tubes is developed. Two small parameters, the ratio of the distance between the tubes to the tube length and the ratio of thickness of regions with varying density to the tube radius, are used to obtain the asymptotic expression for the decrement. This expression is calculated explicitly in a particular case of two identical tubes. The dependence of damping time on the separation distance between the tubes and on the density contrast is investigated. In particular, we obtained that the interaction between the tubes reduces the efficiency of resonant absorption.
\end{abstract}

Key words. magnetohydrodynamics (MHD) - waves - plasmas

\section{Introduction}

The first observations of coronal loop transverse oscillations were made by the transition region and coronal explorer (TRACE) space station on 14 July 1998. The results of these observations were reported by Aschwanden et al. (1999) and Nakariakov et al. (1999). The oscillations were interpreted as fast kink modes of a cylindrical magnetic flux tube. These and later observations also revealed that the loop oscillations are heavily damped (e.g. Aschwanden et al. 2002; Ofman \& Aschwanden 2002; Schrijver et al. 2002). The mechanism responsible for this rapid damping has been the subject of much speculation since the initial observations. A number of different mechanisms have been suggested. At present it seems that the most reliable mechanism able to explain all the observational properties of damping is resonant absorption (Ruderman \& Roberts 2002; Goossens et al. 2002; Andries et al. 2005; Dymova \& Ruderman 2006; Goossens et al. 2006). Recently Morton \& Erdély (2009) showed that the damping of kink oscillations can be also due to quick cooling of oscillating coronal loops.

It is often observed that the excitation and damping of coronal loop oscillations occurs in groups of coronal loops rather than in one isolated loop (e.g. Verwichte et al. 2004). Moreover it has also been suggested that the coronal magnetic loops are in fact collections of very thin magnetic ropes bundled together (e.g. Martens et al. 2002; Schmelz et al. 2003, 2005; Aschwanden 2005; Aschwanden \& Nightingale 2005). It is therefore important to understand how the interaction of these neighbouring loops affects the oscillations and damping of coronal loops. To our knowledge Arregui et al. (2007) were the first who investigated the influence of internal structuring of coronal loops on the properties of damped kink oscillations using Cartesian geometry. Resonantly damped oscillations of a system of two coronal loops, once again in Cartesian geometry, were studied by Arregui et al. (2008).
It is well known that the properties of sausage waves in magnetic slabs and tubes are very similar, while the properties of kink wave are quite different. Hence it is desirable to study the collective kink-like oscillations of systems of coronal loops modeling individual loops as magnetic flux tubes. A natural starting point to approach this problem is studying the collective oscillations of just two coronal loops modelled by two parallel magnetic tubes. This problem was first addressed in the numerical study by Luna et al. (2008). These authors studied the collective kink oscillations of two identical parallel magnetic tubes. They found that there are four modes of oscillations of this system. Two of them are polarized in the direction connecting the loop axes, and two others in the orthogonal direction. In each of the two pairs of modes one corresponds to the tube oscillating in phase, and the other in anti-phase.

Van Doorsselaere et al. (2008) studied the same problem analytically. They relaxed the restriction imposed by Luna et al. (2008) that the tubes are identical and considered the system with different radii of the tubes and different plasma densities inside the tubes. Van Doorsselaere et al. (2008) found that the kink oscillations of the tube system are degenerate, i.e., similar to the kink oscillations of a single tube with the circular cross-section, there are no preferable direction of kink oscillation polarization. They also found that there are only two eigenfrequencies of kink oscillations. All these results are different from those obtained by Luna et al. (2008). Van Doorsselaere et al. (2008) attributed this difference to the fact that they used the long wavelength approximation. As a result two modes with lower frequencies found by Luna et al. (2008) merged in one degenerate mode, and the same occurred with the two modes with the higher frequencies. Since the modes from each pair are polarized in the mutually orthogonal directions, the degenerate modes created by merging of two modes can have arbitrary polarization.

Later the study of collective coronal loop oscillations has been extended in different directions. Luna et al. $(2009,2010)$ studied oscillations of multi-stranded coronal loops and systems 
of many interacting coronal loops using the $T$-matrix theory. Robertson et al. (2010) investigated the effect of density variation along the loop in the two-loop system. The problem of damping of kink oscillations of multi-loop systems has been also addressed. Ofman $(2005,2009)$ studied the damped oscillations of a system of four identical parallel magnetic tubes. Terradas et al. (2008) considered the damping due to resonant absorption of a multi-stranded coronal loop.

The aim of this paper is to study the resonant damping of kink oscillations of a system of two parallel magnetic tubes. In the next section we describe the equilibrium state and write down the governing equations and boundary conditions. In Sect. 3 we study the damped oscillations of the two-tube system. In particular, we derive the expression for the decrement. In Sect. 4 we study the dependence of the decrement on the equilibrium quantities. Section 5 contains the summary of the results obtained in the paper and our conclusions.

\section{Problem formulation}

We model two coronal loops as two straight parallel magnetic cylinders of length $L$ and radii $R_{\mathrm{L}}$ and $R_{\mathrm{R}}$. In Cartesian coordinates $x, y, z$ the loop axes are parallel to the $z$-axis. The equilibrium magnetic field is everywhere homogeneous and in the $z$-direction. Its magnitude is $B$. The $x y$-plane cuts the loops in two equal pats. The magnetic field lines are frozen in the dense photospheric plasma at $z= \pm L / 2$.

In what follows we use bi-cylindrical coordinates $\tau, \sigma, z$. The relation between Cartesian and bi-cylindrical coordinates is given by (e.g. Korn \& Korn 1961)

$x=\frac{a \sinh \tau}{\cosh \tau-\cos \sigma}, \quad y=\frac{a \sin \sigma}{\cosh \tau-\cos \sigma}, \quad z=z$,

where $a$ is a constant. Both $\tau$ and $\sigma$-coordinate lines are circles, all $\tau$-coordinate lines passing through the points $x= \pm a$ on the $x$-axis. The equations of the boundaries of the left and right tube are $\tau=-\tau_{\mathrm{L}}$ and $\tau=\tau_{\mathrm{R}}$ respectively. The tube radii and the distance between their axes, $d$, are expressed in terms of $\tau_{\mathrm{L}}$ and $\tau_{\mathrm{R}}$ by

$R_{\mathrm{L}}=\frac{a}{\sinh \tau_{\mathrm{L}}}, \quad R_{\mathrm{R}}=\frac{a}{\sinh \tau_{\mathrm{R}}}, \quad d=a\left(\operatorname{coth} \tau_{\mathrm{L}}+\operatorname{coth} \tau_{\mathrm{R}}\right)$

The interior of each tube consists of the core region and the annulus attached to the tube boundary. The internal boundary of the left and right annulus are determined by $\tau=-\tau_{\mathrm{L}}-\ell_{\mathrm{L}}$ and $\tau=\tau_{\mathrm{R}}+\ell_{\mathrm{R}}$ respectively. The sketch of the equilibrium is given in Fig. 1.

Outside the tubes the density is $\rho_{\mathrm{e}}$. In the core of the left tube and right tube it is $\rho_{\mathrm{L}}$ and $\rho_{\mathrm{R}}$ respectively, $\rho_{\mathrm{e}}<\rho_{\mathrm{L}, \mathrm{R}}$. In the left and right annulus it decreases from $\rho_{\mathrm{L}}$ to $\rho_{\mathrm{e}}$ and from $\rho_{\mathrm{R}}$ to $\rho_{\mathrm{e}}$. Summarizing, we have

$\rho= \begin{cases}\rho_{\mathrm{L}}, & \tau<-\tau_{\mathrm{L}}-\ell_{\mathrm{L}}, \\ \bar{\rho}_{\mathrm{L}}(\tau), & -\tau_{\mathrm{L}}-\ell_{\mathrm{L}} \leq \tau \leq-\tau_{\mathrm{L}}, \\ \rho_{\mathrm{e}}, & -\tau_{\mathrm{L}}<\tau<\tau_{\mathrm{R}} \\ \bar{\rho}_{\mathrm{R}}(\tau), & \tau_{\mathrm{R}} \leq \tau \leq \tau_{\mathrm{R}}+\ell_{\mathrm{R}} \\ \rho_{\mathrm{R}}, & \tau>\tau_{\mathrm{R}}+\ell_{\mathrm{R}}\end{cases}$

were $\bar{\rho}_{\mathrm{L}}(\tau)$ is monotonically decreasing and $\bar{\rho}_{\mathrm{R}}(\tau)$ monotonically increasing function, $\bar{\rho}_{\mathrm{L}}\left(-\tau_{\mathrm{L}}-\ell_{\mathrm{L}}\right)=\rho_{\mathrm{L}}, \bar{\rho}_{\mathrm{L}}\left(-\tau_{\mathrm{L}}\right)=\rho_{\mathrm{e}}, \bar{\rho}_{\mathrm{R}}\left(\tau_{\mathrm{R}}\right)=$ $\rho_{\mathrm{e}}$, and $\bar{\rho}_{\mathrm{R}}\left(\tau_{\mathrm{L}}+\ell_{\mathrm{R}}\right)=\rho_{\mathrm{R}}$.

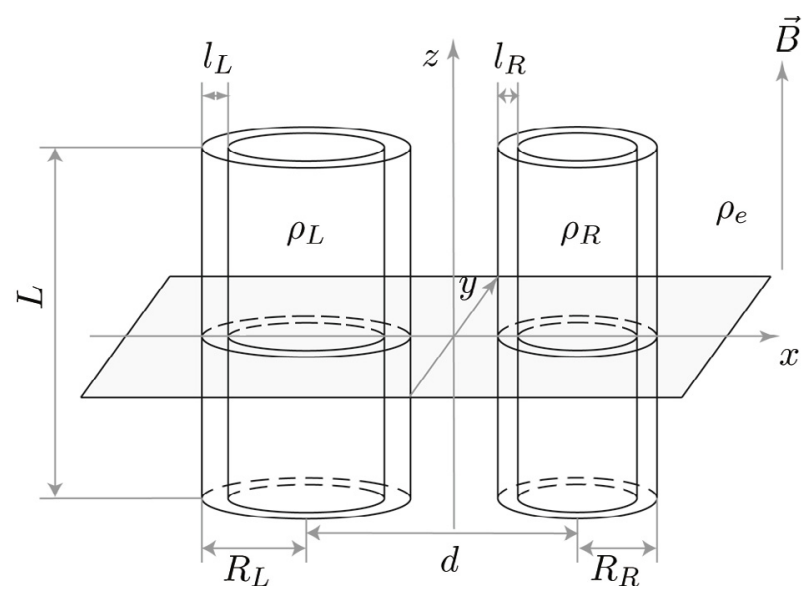

Fig. 1. Sketch of the equilibrium configuration.

In what follows we use the approximation of cold plasma. The plasma motion is described by the system of linearized viscous MHD equations,

$$
\begin{aligned}
& \rho \frac{\partial^{2} \boldsymbol{\xi}}{\partial t^{2}}=\frac{1}{\mu_{0}}(\nabla \times \boldsymbol{b}) \times \boldsymbol{B}+\frac{\partial}{\partial t}[\nabla(\tilde{\eta} \nabla \cdot \boldsymbol{\xi})-\nabla \times(\tilde{\eta} \nabla \times \boldsymbol{\xi})], \\
& \boldsymbol{b}=\nabla \times(\boldsymbol{\xi} \times \boldsymbol{B}) .
\end{aligned}
$$

Here $\boldsymbol{\xi}$ is the plasma displacement, $\boldsymbol{b}$ the magnetic field perturbation, $\boldsymbol{B}=\boldsymbol{B} \boldsymbol{e}_{z}$ the equilibrium magnetic field, $\boldsymbol{e}_{z}$ the unit vector in the $z$-direction, $\mu_{0}$ the magnetic permeability of free space, and $\tilde{\eta}$ the dynamics viscosity. In the solar corona the viscosity tensor is strongly anisotropic, so that, strictly speaking, we have to use the full Braginskii's expression for the viscosity tensor (Braginskii 1965). For typical coronal parameters the coefficient of compressional viscosity in the Braginskii's expression is by many orders of magnitude larger than the coefficient of shear viscosity. However we take viscosity into account only to remove the singularity at the Alfvén resonant position, so that the viscosity is only important in a thin dissipative layer embracing the Alfvén magnetic resonant surface. In spite of the fact that, in general, the compressional viscosity strongly dominates the shear viscosity, it does not affect the plasma motion in the Alfvén dissipative layer (Ofman et al. 1994; Erdélyi \& Goossens 1995). Hence, when writing Eq. (4), we have taken only the shear viscosity into account, and $\tilde{\eta}$ is the coefficient of shear viscosity.

Equations (4) and (5) have to be supplemented by boundary conditions. Since the magnetic field lines are frozen in a dense immovable photospheric plasma at $z= \pm L / 2$, the plasma displacement in the direction orthogonal to the $z$-direction, $\boldsymbol{\xi}_{\perp}$, has to vanish at $z= \pm L / 2$. Hence

$\xi_{\perp}=0 \quad$ at $\quad z= \pm L / 2$.

The system of Eqs. (4) and (5) and the boundary condition (6) will be used in the next section to study the damping of kink oscillations.

\section{Derivation of expression for oscillation decrement}

The aim of this section is to derive the expression for oscillation decrement. To do this we solve the linearized MHD equations separately in the outer region, in the core regions of the magnetic tubes, and in the annuluses, and then match the solutions. 
Let us transform the system of Eqs. (4) and (5). First of all we rewrite these equations as

$\rho \frac{\partial^{2} \boldsymbol{\xi}}{\partial t^{2}}=-\nabla P+\frac{B}{\mu_{0}} \frac{\partial \boldsymbol{b}}{\partial z}+\frac{\partial}{\partial t}[\nabla(\tilde{\eta} \nabla \cdot \boldsymbol{\xi})-\nabla \times(\tilde{\eta} \nabla \times \boldsymbol{\xi})]$,

$\boldsymbol{b}=B \frac{\partial \boldsymbol{\xi}}{\partial z}-\boldsymbol{B} \nabla \cdot \boldsymbol{\xi}$,

where $P=\boldsymbol{b} \cdot \boldsymbol{B} / \mu_{0}$ is the perturbation of magnetic pressure. Viscosity can be neglected everywhere except the dissipative layer where there are large gradients in the $\tau$-direction. This, in particular, implies that we can neglect the derivatives of $\tilde{\eta}$ in comparison with the derivatives of $\boldsymbol{\xi}$. Hence, we can use the approximate relation

$\nabla(\tilde{\eta} \nabla \cdot \boldsymbol{\xi})-\nabla \times(\tilde{\eta} \nabla \times \boldsymbol{\xi}) \approx \tilde{\eta} \nabla^{2} \boldsymbol{\xi}$.

Substituting this relation in Eq. (4) we immediately obtain that $\xi_{z}=0$, i.e. $\boldsymbol{\xi}=\boldsymbol{\xi}_{\perp}$. Taking this result into account, substituting Eq. (8) in (7), and using Eq. (9) we transform Eq. (7) to

$\frac{\partial^{2} \boldsymbol{\xi}}{\partial t^{2}}-V_{\mathrm{A}}^{2} \frac{\partial^{2} \boldsymbol{\xi}}{\partial z^{2}}=-\frac{1}{\rho} \nabla P+\frac{\tilde{\eta}}{\rho} \nabla^{2} \frac{\partial \boldsymbol{\xi}}{\partial t}$,

where $V_{\mathrm{A}}^{2}=B^{2} / \mu_{0} \rho$ is the square of the Alfvén speed. Taking the scalar product of Eq. (8) with $\boldsymbol{B}$ yields

$P=-\rho V_{\mathrm{A}}^{2} \nabla \cdot \boldsymbol{\xi}$.

Since the coefficients in Eqs. (10) and (11) are independent of $z$, we can Fourier-analyze these equations with respect to $z$. In what follows we consider only the fundamental mode with respect to $z$. To satisfy the boundary conditions (6) we take $\xi$ proportional to $\cos (\pi z / L)$. Then it follows from Eq. (11) that $P$ is also proportional to $\cos (\pi z / L)$. We are looking for solutions in the form of dissipative eigenmodes. In accordance with this we take $\boldsymbol{\xi}$ and $P$ proportional to $\exp (-\omega t)$ with complex $\omega$. As a result Eq. (10) reduces to

$\left(\omega^{2}-V_{\mathrm{A}}^{2} k^{2}\right) \boldsymbol{\xi}=\frac{1}{\rho} \nabla P+\frac{\mathrm{i} \omega \tilde{\eta}}{\rho} \nabla^{2} \boldsymbol{\xi}$,

where $k=\pi / L$. Equation (11) remains the same.

Let us now write Eqs. (10), and (11) in bi-cylindrical coordinates $\tau, \sigma, z$. In these coordinates $\boldsymbol{\xi}=\left(\xi_{\tau}, \xi_{\sigma}, 0\right)$,

$$
\begin{aligned}
\nabla f= & \frac{\cosh \tau-\cos \sigma}{a}\left(\boldsymbol{e}_{\tau} \frac{\partial f}{\partial \tau}+\boldsymbol{e}_{\sigma} \frac{\partial f}{\partial \sigma}\right)+\boldsymbol{e}_{z} \frac{\partial f}{\partial z}, \\
\nabla^{2} f= & \frac{(\cosh \tau-\cos \sigma)^{2}}{a^{2}}\left(\frac{\partial^{2} f}{\partial \tau^{2}}+\frac{\partial^{2} f}{\partial \sigma^{2}}\right)+\frac{\partial^{2} f}{\partial z^{2}} \\
\nabla \cdot \boldsymbol{g}= & \frac{(\cosh \tau-\cos \sigma)^{2}}{a}\left[\frac{\partial}{\partial \tau}\left(\frac{g_{\tau}}{\cosh \tau-\cos \sigma}\right)\right. \\
& \left.+\frac{\partial}{\partial \sigma}\left(\frac{g_{\sigma}}{\cosh \tau-\cos \sigma}\right)\right]+\frac{\partial g_{z}}{\partial z} \\
\nabla \times \boldsymbol{g}= & \boldsymbol{e}_{\tau}\left(\frac{\cosh \tau-\cos \sigma}{a} \frac{\partial g_{z}}{\partial \sigma}-\frac{\partial g_{\sigma}}{\partial z}\right) \\
& -\boldsymbol{e}_{\sigma}\left(\frac{\cosh \tau-\cos \sigma}{a} \frac{\partial g_{z}}{\partial \tau}-\frac{\partial g_{\tau}}{\partial z}\right) \\
& +\boldsymbol{e}_{z} \frac{(\cosh \tau-\cos \sigma)^{2}}{a}\left[\frac{\partial}{\partial \tau}\left(\frac{g_{\sigma}}{\cosh \tau-\cos \sigma}\right)\right. \\
& \left.-\frac{\partial}{\partial \sigma}\left(\frac{g_{\tau}}{\cosh \tau-\cos \sigma}\right)\right]
\end{aligned}
$$

(see, e.g., Korn \& Korn 1961). Here $f$ and $\boldsymbol{g}$ are arbitrary scalar and vector-function respectively, and $\boldsymbol{e}_{\tau}$ and $\boldsymbol{e}_{\sigma}$ are the unit vectors in the $\tau$ and $\sigma$ directions respectively. Using the identity $\nabla^{2} \boldsymbol{\xi}=\nabla \nabla \cdot \boldsymbol{\xi}-\nabla \times \nabla \times \boldsymbol{\xi}$ and Eqs. (13), (15) and (16) we can easily obtain the expression for $\nabla^{2} \boldsymbol{\xi}$. Repeat that viscosity is only important in a thin dissipative layer embracing the resonant magnetic surface. In this layer there are large gradients only in the $\tau$-direction. This observation enables us to neglect all terms in the expression for $\nabla^{2} \boldsymbol{\xi}$ but the term proportional to the second derivative of $\boldsymbol{\xi}$ with respect to $\tau$. As a result we arrive at the approximate expression

$\nabla^{2} \boldsymbol{\xi} \approx \frac{(\cosh \tau-\cos \sigma)^{2}}{a^{2}} \frac{\partial^{2} \xi}{\partial \tau^{2}}$

In the limit of very small viscosity the damping rate of oscillations due to resonant absorption is independent of the particular value of $\tilde{\eta}$ (e.g. Goossens \& Ruderman 1995). In particular, we can assume any dependence of $\tilde{\eta}$ on $\tau$ and $\sigma$ under the only restriction that the characteristic scale of variation of $\tilde{\eta}$ is much larger than the thickness of the dissipative layer. To simplify the calculations as much as possible we take

$\tilde{\eta}=\frac{\rho(\tau) v}{(\cosh \tau-\cos \sigma)^{2}}$,

where $v$ is a constant. Now we can write the system of Eqs. (11) and (12) in components,

$$
\begin{aligned}
& P=-\frac{1}{a} \rho V_{\mathrm{A}}^{2}(\cosh \tau-\cos \sigma)^{2} \\
& \times\left[\frac{\partial}{\partial \tau}\left(\frac{\boldsymbol{\xi}_{\tau}}{\cosh \tau-\cos \sigma}\right)+\frac{\partial}{\partial \sigma}\left(\frac{\boldsymbol{\xi}_{\sigma}}{\cosh \tau-\cos \sigma}\right)\right], \\
&\left(\omega^{2}-V_{\mathrm{A}}^{2} k^{2}\right) \xi_{\tau}=\frac{\cosh \tau-\cos \sigma}{a \rho} \frac{\partial P}{\partial \tau}+\frac{\mathrm{i} \omega \nu}{a^{2}} \frac{\partial^{2} \xi_{\tau}}{\partial \tau^{2}} \\
&\left(\omega^{2}-V_{\mathrm{A}}^{2} k^{2}\right) \xi_{\sigma}=\frac{\cosh \tau-\cos \sigma}{a \rho} \frac{\partial P}{\partial \sigma}+\frac{\mathrm{i} \omega \nu}{a^{2}} \frac{\partial^{2} \xi_{\sigma}}{\partial \tau^{2}}
\end{aligned}
$$

These equations will be used in the next subsections to derive the expression for the decrement.

\subsection{Solution in the outer and core regions}

In the outer and core regions we neglect viscosity. The density in these regions is constant. Eliminating $\xi_{\tau}$ and $\xi_{\sigma}$ from Eqs. (19)(21) with the terms describing viscosity neglected we obtain the equation for $P$,

$\frac{\partial^{2} P}{\partial \tau^{2}}+\frac{\partial^{2} P}{\partial \sigma^{2}}+\frac{a^{2}\left(\omega^{2}-V_{\mathrm{A}}^{2} k^{2}\right) P}{V_{\mathrm{A}}^{2}(\cosh \tau-\cos \sigma)^{2}}=0$.

In what follows we assume that the tube length $\mathrm{L}$ is much larger than the characteristic size of the system in the directions orthogonal to the tube axes. We can take this characteristic size equal to $d$. Hence, we assume that $d / L=\epsilon \ll 1$. Now we easily obtain that, since $\omega \sim k V_{\mathrm{A}}$, the ratio of the last term in Eq. (22) to the first and second term is of the order of $\epsilon^{2}$. Neglecting this small term we obtain the approximate equation for $P$,

$\frac{\partial^{2} P}{\partial \tau^{2}}+\frac{\partial^{2} P}{\partial \sigma^{2}}=0$

It follows from Eq. (1) that $\tau^{2}+\sigma^{2} \rightarrow 0$ far from the magnetic tubes, i.e. when $x^{2}+y^{2} \rightarrow \infty$. The general solution to Eq. (23) 
can be looked for in the form of Fourier expansion with respect to $\sigma$. However we are only interested in the kink oscillations, so that we look for the solution to Eq. (23) in the form

$P=\Phi(\tau) \cos \left(\sigma-\sigma_{0}\right)-\Phi(0) \cos \sigma_{0}$,

where $\sigma_{0}$ is a constant and $\Phi(\tau)$ a function to be determined. The second term on the right-hand side of this expression is introduced to satisfy the condition that $P \rightarrow 0$ as $\tau^{2}+\sigma^{2} \rightarrow 0$. When $\tau \rightarrow \pm \infty$ we have $x= \pm a$ and $y \rightarrow 0$. This implies that the solution to Eq. (23) has to be bounded when $\tau \rightarrow \pm \infty$. Then we immediately obtain that

$\Phi(\tau)=\left\{\begin{array}{lr}C_{\mathrm{L}} \mathrm{e}^{\tau}, & \tau \leq-\tau_{\mathrm{L}}-l_{\mathrm{L}}, \\ C_{1} \mathrm{e}^{\tau}+C_{2} \mathrm{e}^{-\tau}, & -\tau_{\mathrm{L}} \leq \tau \leq \tau_{\mathrm{R}}, \\ C_{\mathrm{R}} \mathrm{e}^{-\tau}, & \tau \geq \tau_{\mathrm{R}}+l_{\mathrm{R}},\end{array}\right.$

where $C_{1}, C_{2}, C_{\mathrm{L}}$ and $C_{\mathrm{R}}$ are arbitrary constants. Now, using Eq. (20) with the last term neglected, we arrive at

$\xi_{\tau}=\hat{\xi}_{\tau}(\tau)(\cosh \tau-\cos \sigma) \cos \left(\sigma-\sigma_{0}\right)$

where

$\hat{\xi}_{\tau}= \begin{cases}\frac{C_{\mathrm{L}} \mathrm{e}^{\tau}}{a \rho_{\mathrm{L}}\left(\omega^{2}-V_{\mathrm{AL}}^{2} k^{2}\right)}, & \tau \leq-\tau_{\mathrm{L}}-l_{\mathrm{L}}, \\ \frac{C_{1} \mathrm{e}^{\tau}-C_{2} \mathrm{e}^{-\tau}}{a \rho_{\mathrm{e}}\left(\omega^{2}-V_{\mathrm{Ae}}^{2} k^{2}\right)}, & -\tau_{\mathrm{L}} \leq \tau \leq \tau_{\mathrm{R}}, \\ \frac{-C_{\mathrm{R}} \mathrm{e}^{-\tau}}{a \rho_{\mathrm{R}}\left(\omega^{2}-V_{\mathrm{AR}}^{2} k^{2}\right)}, & \tau \geq \tau_{\mathrm{R}}+l_{\mathrm{R}} .\end{cases}$

Since $P$ and $\xi$ has to be continuous at the annulus boundaries, Eq. (24) is also valid in the annuluses. Then it follows from Eq. (20) that Eq. (26) is also valid in the annuluses. Finally, it follows from Eq. (20) that we can write $\xi_{\sigma}$ as

$\xi_{\sigma}=-\hat{\xi}_{\sigma}(\tau)(\cosh \tau-\cos \sigma) \sin \left(\sigma-\sigma_{0}\right)$.

Substituting Eqs. (26) and (28) in Eq. (19) we obtain

$$
\left(\frac{\mathrm{d} \hat{\xi}_{\tau}}{\mathrm{d} \tau}-\hat{\xi}_{\sigma}\right) \cos \left(\sigma-\sigma_{0}\right)=-\frac{a P}{\rho V_{\mathrm{A}}^{2}(\cosh \tau-\cos \sigma)^{2}} .
$$

It is easy to see that this equation is inconsistent with Eq. (24). However let us estimate the order of terms in Eq. (29). It follows from Eq. (21) that $P \sim a k^{2} \rho V_{\mathrm{A}}^{2} \xi_{\sigma} \sim \epsilon k \rho V_{\mathrm{A}}^{2} \hat{\xi}_{\sigma}$. Then it immediately follows that the ratio of the right-hand side of Eq. (29) to its left-hand side is of the order of $\epsilon^{2}$, so that the right-hand side can be neglected in the long wavelength approximation. As a result Eq. (29) is simplified to

$\frac{\mathrm{d} \hat{\xi}_{\tau}}{\mathrm{d} \tau}-\hat{\xi}_{\sigma}=0$

Obviously this equation does not contradict to Eq. (24). This analysis clearly shows that the expressions for $P$ and $\boldsymbol{\xi}$ given by Eqs. (24), (26) and (28) can be used only in the long wavelength approximation.

\subsection{Solution in the dissipative layer}

When the tubes are homogeneous Van Doorsselaere et al. (2008) has shown that, in the long wavelength approximation, there are two eigenfrequencies of kink modes, $\omega_{-}$and $\omega_{+}, \omega_{-}<\omega_{+}$. All systems of two parallel magnetic tubes can be separated in two classes: standard and anomalous. In standard systems

$k V_{\mathrm{AL}, \mathrm{R}}<\omega_{ \pm}<\omega_{\mathrm{e}}$.

In anomalous systems

$k V_{\mathrm{AL}, \mathrm{R}}<\omega_{+}<\omega_{\mathrm{e}}$,

$k \min \left(V_{\mathrm{AL}}, V_{\mathrm{AR}}\right)<\omega_{-}<k \max \left(V_{\mathrm{AL}}, V_{\mathrm{AR}}\right)$.

In what follows assume that the annuluses are thin, $\ell_{\mathrm{L}} \ll R_{\mathrm{L}}$ and $\ell_{\mathrm{R}} \ll R_{\mathrm{R}}$. In that case the main effect due to the presence of the annuluses is the resonant damping of a kink mode, the real part of the frequency only being slightly modified. Hence, the real part of eigenfrequency of a damped dissipative eigenmode is approximately equal to either $\omega_{-}$or $\omega_{+}$. The resonance conditions for the left and right tubes are written as

$k V_{\mathrm{A}}\left(\tau_{\mathrm{AL}}\right)=\omega, \quad k V_{\mathrm{A}}\left(\tau_{\mathrm{AR}}\right)=\omega$,

where $\tau=\tau_{\mathrm{AL}}$ and $\tau=\tau_{\mathrm{AR}}$ are the equations of the resonant surfaces in the right and left annulus respectively, and $\omega$ is equal either $\omega_{-}$or $\omega_{+}$. Then it follows from Eq. (31) that, in a standard system, there is the resonant surface both in the left and right annulus for both the slower $\left(\omega=\omega_{-}\right)$and faster $\left(\omega=\omega_{+}\right)$eigenmodes. On the other hand, it follows from Eq. (32) that, in an anomalous system, there is the resonant surface both in the left and right annulus only for the faster eigenmode. For the slower eigenmode there is only one resonant surface. This surface is in the denser tube, i.e. in the tube with the smaller Alfvén speed in the core region.

Let us study the wave motion in the dissipative layer embracing a resonant surface. The analysis is the same for the dissipative layers in the left and right tubes, so that we will drop the subscripts "L" and " $\mathrm{R}$ " in what follows.

Substituting Eqs. (24), (26) and (28) in Eqs. (20) and (21) we obtain

$\left(\omega^{2}-V_{\mathrm{A}}^{2} k^{2}\right) \hat{\xi}_{\tau}-\frac{\mathrm{i} \omega \nu}{a^{2}} \frac{\mathrm{d}^{2} \hat{\xi}_{\tau}}{\mathrm{d} \tau^{2}}=\frac{1}{a \rho} \frac{\mathrm{d} \Phi}{\mathrm{d} \tau}$

$\left(\omega^{2}-V_{\mathrm{A}}^{2} k^{2}\right) \hat{\xi}_{\sigma}-\frac{\mathrm{i} \omega \nu}{a^{2}} \frac{\mathrm{d}^{2} \hat{\xi}_{\sigma}}{\mathrm{d} \tau^{2}}=\frac{\Phi}{a \rho}$.

In what follows we will see that the ration of the decrement $\gamma$ to the real part of the eigenfrequency is of the order of $\ell / R \ll 1$. This implies that we can use the approximation

$\omega^{2} \approx \omega_{0}^{2}-2 \mathrm{i} \omega_{0} \gamma$

where either $\omega_{0}=\omega_{-}$or $\omega_{0}=\omega_{+}$. We also can substitute $\omega_{0}$ for $\omega$ in the coefficient at the second derivative in Eqs. (34) and (35). The equation of the resonant magnetic surface is $\tau=\tau_{\mathrm{A}}$, where $\tau_{\mathrm{A}}$ is defined by $k V_{\mathrm{A}}\left(\tau_{\mathrm{A}}\right)=\omega_{0}$. The characteristic scale of variation of $V_{\mathrm{A}}$ in the annulus is $\ell$, while the thickness of the dissipative layer is much smaller than $\ell$. This implies that, in the dissipative layer we can use the approximation

$V_{\mathrm{A}}^{2} k^{2} \approx \omega_{0}^{2}-\Delta\left(\tau-\tau_{\mathrm{A}}\right), \quad \Delta=-\left.k^{2} \frac{\mathrm{d} V_{\mathrm{A}}^{2}}{\mathrm{~d} \tau}\right|_{\tau=\tau_{\mathrm{A}}}$

It is convenient to introduce the new variable

$s=\frac{\tau-\tau_{\mathrm{A}}}{\delta}, \quad \delta=\left(\frac{\omega_{0} v}{a^{2}|\Delta|}\right)^{1 / 3}$. 
Note that $\delta \sim R_{\mathrm{e}}^{-1 / 3}\left(\ell L^{2} / a^{2}\right)^{1 / 3}$, where $R_{\mathrm{e}}=\omega_{0} L^{2} / v$ is the Reynolds number. For typical coronal conditions $R_{\mathrm{e}}$ is so large that $\delta \ll 1$.

Using Eqs. (36)-(38) we rewrite Eqs. (30), (34) and (35) in the new variables as

$\frac{\mathrm{d} \hat{\xi}_{\tau}}{\mathrm{d} s}-\delta \hat{\xi}_{\sigma}=0$

$\frac{\mathrm{d}^{2} \hat{\xi}_{\tau}}{\mathrm{d} s^{2}}+(\mathrm{i} s \operatorname{sgn}(\Delta)+\Lambda) \hat{\xi}_{\tau}=\frac{\mathrm{i}}{a \rho_{\mathrm{A}}|\Delta| \delta^{2}} \frac{\mathrm{d} \Phi}{\mathrm{d} s}$,

$\frac{\mathrm{d}^{2} \hat{\xi}_{\sigma}}{\mathrm{d} s^{2}}+(\mathrm{i} s \operatorname{sgn}(\Delta)+\Lambda) \hat{\xi}_{\sigma}=\frac{\mathrm{i} \Phi}{a \rho_{\mathrm{A}}|\Delta| \delta}$,

where $\Lambda=2 \omega_{0} \gamma / \delta|\Delta|$ and $\rho_{\mathrm{A}}$ is the density calculated at the resonant position, i.e. $\rho_{\mathrm{A}}=\bar{\rho}\left(\tau_{\mathrm{A}}\right)$. Now we differentiate Eq. (40), subtract Eq. (41) multiplied by $\delta$ from the result, and use Eq. (39) to obtain

$\frac{\mathrm{d}^{2} \Phi}{\mathrm{d} s^{2}}=\delta^{2}\left(\Phi+a \rho_{\mathrm{A}} \Delta \hat{\xi}_{\tau}\right)$

Since $\delta \ll 1$ it follows from this equation that the variation of $\Phi$ in the dissipative layer is very small, and we can take $\Phi \approx$ const. $=\Phi\left(\tau_{\mathrm{A}}\right)$ in the dissipative layer. This result is well known in the theory of resonant MHD waves in planar and cylindrical geometry (e.g. Hollweg 1987; Goossens at al. 1995). With $\Phi=$ const. Eq. (41) coincides, with the accuracy up to the notation, with the corresponding equation considered by Tirry \& Goossens (1996). Hence, we can us the results obtained by these authors and immediately write

$\hat{\xi}_{\sigma}=-\frac{\mathrm{i} \Phi\left(\tau_{\mathrm{A}}\right)}{a \rho_{\mathrm{A}}|\Delta| \delta} F_{\Lambda}(s)$,

where

$\left.F_{\Lambda}(s)=\int_{0}^{\infty} \exp \left\{\mathrm{i} u s \operatorname{sgn}(\Delta)-u^{3} / 3+\Lambda u\right)\right\} \mathrm{d} u$.

Substituting Eq. (43) in (39) and using Eq. (44) we obtain

$\hat{\xi}_{\tau}=-\frac{\Phi\left(\tau_{\mathrm{A}}\right)}{a \rho_{\mathrm{A}} \Delta} G_{\Lambda}(s)+$ const

where

$G_{\Lambda}(s)=\int_{0}^{\infty} \frac{\mathrm{e}^{-u^{3} / 3}}{u}\{\exp (\mathrm{i} u s \operatorname{sgn}(\Delta)+\Lambda u)-1\} \mathrm{d} u$.

The functions $F_{\Lambda}$ and $G_{\Lambda}$ were first introduced by Ruderman at al. (1995).

Let us introduce the jump of a function $f(s)$ across the dissipative layer,

$[f]=\lim _{s \rightarrow \infty}\{f(s)-f(-s)\}$.

Since we take $\Phi=$ const. in the dissipative layer we immediately obtain

$[\Phi]=0$.

It is straightforward to obtain $[G]=\mathrm{i} \pi \operatorname{sgn}(\Delta)$. It follows from this result and Eq. (45) that

$\left[\hat{\xi}_{\tau}\right]=-\frac{\mathrm{i} \pi \Phi\left(\tau_{\mathrm{A}}\right)}{a \rho_{\mathrm{A}}|\Delta|}$.

Equations (47) and (48) will be used in the next subsection to connect the solutions at the left and the right of the dissipative layer.

\subsection{Variation of pressure and velocity across the annuluses}

In this subsections we calculate the variations of $P$ and $\hat{\xi}_{\tau}$ across the annuluses. We give the detailed calculation of these variations in the left annulus, and then present only the final results for the right annulus. Since we assume that $\ell \ll 1$, in our calculations we will keep only the linear terms with respect to $\ell$, while we will neglect quadratic terms and terms of the higher order.

To calculate the variation of $\hat{\xi}_{\tau}$ across the annuluses we use Eqs. (30) and (35). Since Eq. (35) is used outside the dissipative layer, we neglect the last term on its right-hand side. Since, in accordance with Eq. (47) $\Phi$ does not jump across the dissipative layer, and we neglect the variation of $\Phi$ when calculating $\hat{\xi}_{\tau}$, we take $\Phi=\Phi\left(\tau_{\mathrm{AL}}\right) \equiv \Phi_{\mathrm{AL}}$ in Eq. (35) (recall that $\tau=\tau_{\mathrm{AL}}$ is the equation of the resonant magnetic surface in the left annulus). Since the imaginary part of $\omega$ is of the order of $\ell$ we also substitute $\omega_{0}$ for $\omega$. Then we easily obtain

$\hat{\xi}_{\tau}=\hat{\xi}_{\tau}\left(-\tau_{\mathrm{L}}-\ell_{\mathrm{L}}\right)+\frac{\Phi_{\mathrm{AL}}}{a} \int_{-\tau_{\mathrm{L}}-\ell_{\mathrm{L}}}^{\tau} \frac{\mathrm{d} \tilde{\tau}}{\bar{\rho}_{\mathrm{L}}(\tilde{\tau})\left[\omega_{0}^{2}-k^{2} V_{\mathrm{A}}^{2}(\tilde{\tau})\right]}$,

for $-\tau_{\mathrm{L}}-\ell_{\mathrm{L}} \leq \tau<\tau_{\mathrm{AL}}$, and

$\hat{\xi}_{\tau}=\hat{\xi}_{\tau}\left(-\tau_{\mathrm{L}}\right)-\frac{\Phi_{\mathrm{AL}}}{a} \int_{\tau}^{-\tau_{\mathrm{L}}} \frac{\mathrm{d} \tilde{\tau}}{\bar{\rho}_{\mathrm{L}}(\tilde{\tau})\left[\omega_{0}^{2}-k^{2} V_{\mathrm{A}}^{2}(\tilde{\tau})\right]}$,

for $\tau_{\mathrm{AL}}<\tau<\ell_{\mathrm{L}}-\tau_{\mathrm{L}}$. Equations (49) and (50) clearly show that $\hat{\xi}_{\tau}$ has a logarithmic singularity at $\tau=\tau_{\mathrm{AL}}$ in a complete agreement with the general theory of resonant MHD waves.

Equations (49) and (50) give the ideal solution outside the dissipative layer in the left annulus. Now we match this solution with the solution in the dissipative layer in two overlap layers at the left and right of the dissipative layer. In these layers both the ideal and dissipative solution are valid. It follows from the matching condition that the limit of any quantity defined in the dissipative layer as $s \rightarrow \pm \infty$ has to coincide with the limit of the same quantity defined outside the dissipative layer as $\tau \rightarrow \pm 0$. It follows from this condition that we can give another formula for the jump of function $f$ across the dissipative layer,

$[f]=\lim _{\varepsilon \rightarrow+0}\left\{f\left(\tau_{\mathrm{AL}}+\varepsilon\right)-f\left(\tau_{\mathrm{AL}}-\varepsilon\right)\right\}$.

Then we obtain from Eqs. (49) and (50) that

$\left[\hat{\xi}_{\tau}\right]=\delta \hat{\xi}_{\tau \mathrm{L}}-\frac{\Phi_{\mathrm{AL}}}{a} \mathcal{P} \int_{-\tau_{\mathrm{L}}-\ell_{\mathrm{L}}}^{-\tau_{\mathrm{L}}} \frac{\mathrm{d} \tau}{\bar{\rho}_{\mathrm{L}}(\tau)\left[\omega_{0}^{2}-k^{2} V_{\mathrm{A}}^{2}(\tau)\right]}$

where $\mathcal{P}$ indicates the principal Cauchy part of the integral, and

$\delta \hat{\xi}_{\tau \mathrm{L}}=\hat{\xi}_{\tau}\left(-\tau_{\mathrm{L}}\right)-\hat{\xi}_{\tau}\left(-\tau_{\mathrm{L}}-\ell_{\mathrm{L}}\right)$.

Comparing Eqs. (48) and (51) yields

$\delta \hat{\xi}_{\tau \mathrm{L}}=\frac{\Phi_{\mathrm{AL}}}{a}\left(\mathcal{P} \int_{-\tau_{\mathrm{L}}-\ell_{\mathrm{L}}}^{-\tau_{\mathrm{L}}} \frac{\mathrm{d} \tau}{\bar{\rho}_{\mathrm{L}}(\tau)\left[\omega_{0}^{2}-k^{2} V_{\mathrm{A}}^{2}(\tau)\right]}-\frac{\mathrm{i} \pi}{\rho_{\mathrm{AL}}\left|\Delta_{\mathrm{L}}\right|}\right)$

To calculate the variation of $\Phi$ across the annulus we use Eq. (34) with the last term on the left-hand side neglected and $\omega_{0}$ substituted for $\omega$. Since, in accordance with Eq. (53) the variation of $\hat{\xi}_{\tau}$ across the annulus is of the order of $\ell_{\mathrm{L}}$, we neglect it when 
calculating the variation of $\Phi$ and substitute $\hat{\xi}_{\tau}\left(-\tau_{L}\right)$ for $\hat{\xi}_{\tau}$ in Eq. (34). Then we immediately obtain

$\delta \Phi_{\mathrm{L}}=a \hat{\xi}_{\tau}\left(-\tau_{\mathrm{L}}\right) \int_{-\tau_{\mathrm{L}}-\ell_{\mathrm{L}}}^{-\tau_{\mathrm{L}}} \bar{\rho}_{\mathrm{L}}(\tau)\left[\omega_{0}^{2}-k^{2} V_{\mathrm{A}}^{2}(\tau)\right] \mathrm{d} \tau$,

where

$\delta \Phi_{\mathrm{L}}=\Phi\left(-\tau_{\mathrm{L}}\right)-\Phi\left(-\tau_{\mathrm{L}}-\ell_{\mathrm{L}}\right)$.

Repeating the same calculation but for the right annulus yields

$\delta \hat{\xi}_{\tau \mathrm{R}}=\frac{\Phi_{\mathrm{AR}}}{a}\left(\frac{\mathrm{i} \pi}{\rho_{\mathrm{AR}}\left|\Delta_{\mathrm{R}}\right|}-\mathcal{P} \int_{\tau_{\mathrm{R}}}^{\tau_{\mathrm{R}}+\ell_{\mathrm{R}}} \frac{\mathrm{d} \tau}{\bar{\rho}_{\mathrm{R}}(\tau)\left[\omega_{0}^{2}-k^{2} V_{\mathrm{A}}^{2}(\tau)\right]}\right)$,

$\delta \Phi_{\mathrm{R}}=-a \hat{\xi}_{\tau}\left(\tau_{\mathrm{R}}\right) \int_{\tau_{\mathrm{R}}}^{\tau_{\mathrm{R}}+\ell_{\mathrm{R}}} \bar{\rho}_{\mathrm{R}}(\tau)\left[\omega_{0}^{2}-k^{2} V_{\mathrm{A}}^{2}(\tau)\right] \mathrm{d} \tau$

where

$\delta \hat{\xi}_{\tau \mathrm{R}}=\hat{\xi}_{\tau}\left(\tau_{\mathrm{R}}\right)-\hat{\xi}_{\tau}\left(\tau_{\mathrm{R}}+\ell_{\mathrm{R}}\right), \delta \Phi_{\mathrm{R}}=\Phi\left(\tau_{\mathrm{R}}\right)-\Phi\left(\tau_{\mathrm{R}}+\ell_{\mathrm{R}}\right)$

\subsection{Matching solutions and calculation of decrements}

Now we match the solutions in the core regions with the solution outside the tubes. For this we calculate the variations of $\delta \hat{\xi}_{\tau}$ and $\Phi$ across the annuluses using Eqs. (25) and (27), and then compare them with those given by Eqs. (52)-(58). As a result we obtain

$$
\begin{aligned}
C_{1} \mathrm{e}^{-\tau_{\mathrm{L}}}+C_{2} \mathrm{e}^{\tau_{\mathrm{L}}} & -C_{\mathrm{L}} \mathrm{e}^{-\tau_{\mathrm{L}}-\ell_{\mathrm{L}}}=\frac{C_{1} \mathrm{e}^{-\tau_{\mathrm{L}}}-C_{2} \mathrm{e}^{\tau_{\mathrm{L}}}}{\rho_{\mathrm{e}}\left(\omega_{0}^{2}-V_{\mathrm{Ae}}^{2} k^{2}\right)} \\
& \times \int_{-\tau_{\mathrm{L}}-\ell_{\mathrm{L}}}^{-\tau_{\mathrm{L}}} \bar{\rho}_{\mathrm{L}}(\tau)\left[\omega_{0}^{2}-k^{2} V_{\mathrm{A}}^{2}(\tau)\right] \mathrm{d} \tau, \\
C_{1} \mathrm{e}^{\tau_{\mathrm{R}}}+C_{2} \mathrm{e}^{-\tau_{\mathrm{R}}} & -C_{\mathrm{R}} \mathrm{e}^{-\tau_{\mathrm{R}}-\ell_{\mathrm{R}}}=\frac{C_{1} \mathrm{e}^{\tau_{\mathrm{R}}}-C_{2} \mathrm{e}^{-\tau_{\mathrm{R}}}}{\rho_{\mathrm{e}}\left(\omega_{0}^{2}-V_{\mathrm{Ae}}^{2} k^{2}\right)} \\
& \times \int_{\tau_{\mathrm{R}}}^{\tau_{\mathrm{R}}+\ell_{\mathrm{R}}} \bar{\rho}_{\mathrm{L}}(\tau)\left[\omega_{0}^{2}-k^{2} V_{\mathrm{A}}^{2}(\tau)\right] \mathrm{d} \tau,
\end{aligned}
$$

$$
\begin{array}{r}
\frac{C_{1} \mathrm{e}^{-\tau_{\mathrm{L}}}-C_{2} \mathrm{e}^{\tau_{\mathrm{L}}}}{\rho_{\mathrm{e}}\left(\omega^{2}-V_{\mathrm{Ae}}^{2} k^{2}\right)}-\frac{C_{\mathrm{L}} \mathrm{e}^{-\tau_{\mathrm{L}}-\ell_{\mathrm{L}}}}{\rho_{\mathrm{L}}\left(\omega^{2}-V_{\mathrm{AL}}^{2} k^{2}\right)}=\left(C_{1} \mathrm{e}^{-\tau_{\mathrm{L}}}+C_{2} \mathrm{e}^{\tau_{\mathrm{L}}}\right) \\
\times\left(\mathcal{P} \int_{-\tau_{\mathrm{L}}-\ell_{\mathrm{L}}}^{-\tau_{\mathrm{L}}} \frac{\mathrm{d} \tau}{\bar{\rho}_{\mathrm{L}}(\tau)\left[\omega_{0}^{2}-k^{2} V_{\mathrm{A}}^{2}(\tau)\right]}-\frac{\mathrm{i} \pi}{\rho_{\mathrm{AL}}\left|\Delta_{\mathrm{L}}\right|}\right),
\end{array}
$$

$$
\begin{array}{r}
\frac{C_{1} \mathrm{e}^{\tau_{\mathrm{R}}}-C_{2} \mathrm{e}^{-\tau_{\mathrm{R}}}}{\rho_{\mathrm{e}}\left(\omega^{2}-V_{\mathrm{Ae}}^{2} k^{2}\right)}+\frac{C_{\mathrm{R}} \mathrm{e}^{-\tau_{\mathrm{R}}-\ell_{\mathrm{R}}}}{\rho_{\mathrm{R}}\left(\omega^{2}-V_{\mathrm{AR}}^{2} k^{2}\right)}=\left(C_{1} \mathrm{e}^{\tau_{\mathrm{R}}}+C_{2} \mathrm{e}^{-\tau_{\mathrm{R}}}\right) \\
\quad \times\left(\frac{\mathrm{i} \pi}{\rho_{\mathrm{AR}}\left|\Delta_{\mathrm{R}}\right|}-\mathcal{P} \int_{\tau_{\mathrm{R}}}^{\tau_{\mathrm{R}}+\ell_{\mathrm{R}}} \frac{\mathrm{d} \tau}{\bar{\rho}_{\mathrm{R}}(\tau)\left[\omega_{0}^{2}-k^{2} V_{\mathrm{A}}^{2}(\tau)\right]}\right)
\end{array}
$$

When deriving Eqs. (59)-(62) we have used the formulae obtained with the aid of Eqs. (25) and (27),

$$
\begin{aligned}
& \Phi_{\mathrm{AL}} \approx \Phi\left(-\tau_{\mathrm{L}}\right)=C_{1} \mathrm{e}^{-\tau_{\mathrm{L}}}+C_{2} \mathrm{e}^{\tau_{\mathrm{L}}}, \\
& \Phi_{\mathrm{AR}} \approx \Phi\left(\tau_{\mathrm{R}}\right)=C_{1} \mathrm{e}^{\tau_{\mathrm{R}}}+C_{2} \mathrm{e}^{-\tau_{\mathrm{R}}}, \\
& \hat{\xi}_{\tau}\left(-\tau_{\mathrm{L}}\right) \approx \frac{C_{1} \mathrm{e}^{-\tau_{\mathrm{L}}}-C_{2} \mathrm{e}^{\tau_{\mathrm{L}}}}{a \rho_{\mathrm{e}}\left(\omega_{0}^{2}-V_{\mathrm{Ae}}^{2} k^{2}\right)}, \quad \hat{\xi}_{\tau}\left(\tau_{\mathrm{R}}\right) \approx \frac{C_{1} \mathrm{e}^{\tau_{\mathrm{R}}}-C_{2} \mathrm{e}^{-\tau_{\mathrm{R}}}}{a \rho_{\mathrm{e}}\left(\omega_{0}^{2}-V_{\mathrm{Ae}}^{2} k^{2}\right)} .
\end{aligned}
$$

The system of Eqs. (59)-(62) is the system of linear homogeneous algebraic equations. It has a nontrivial solution only if its determinant is zero. This condition gives the dispersion equation that relates $\omega$ and $k$.

The ratios of the right-hand sides of Eqs. (59)-(62) to their left-hand sides are of the order of either $\ell_{\mathrm{L}} \ll 1$ or $\ell_{\mathrm{R}} \ll 1$. This observation enables us to use the regular perturbation method to solve the system of Eqs. (59)-(62). In accordance with this method we look for the solution in the form

$C_{1}=C_{10}+C_{11}, \quad C_{2}=C_{20}+C_{21}$,

$C_{\mathrm{L}}=C_{\mathrm{L} 0}+C_{\mathrm{L} 1}, \quad C_{\mathrm{R}}=C_{\mathrm{R} 0}+C_{\mathrm{R} 1}$,

where the second terms on the right-hand sides of these expressions are much smaller than the first terms. In addition, we write

$\omega=\omega_{0}+\omega_{1}$,

where $\left|\omega_{1}\right| \ll\left|\omega_{0}\right|$.

\subsubsection{Zero-order approximation}

Substituting Eqs. (64) and (65) in Eqs. (59)-(62), keeping only the largest terms and neglecting the right-hand sides we obtain in the zero-order approximation

$$
\begin{aligned}
& C_{10} \mathrm{e}^{-\tau_{\mathrm{L}}}+C_{20} \mathrm{e}^{\tau_{\mathrm{L}}}-C_{\mathrm{L} 0} \mathrm{e}^{-\tau_{\mathrm{L}}}=0, \\
& C_{10} \mathrm{e}^{\tau_{\mathrm{R}}}+C_{20} \mathrm{e}^{-\tau_{\mathrm{R}}}-C_{\mathrm{R} 0} \mathrm{e}^{-\tau_{\mathrm{R}}}=0, \\
& \frac{C_{10} \mathrm{e}^{-\tau_{\mathrm{L}}}-C_{20} \mathrm{e}^{\tau_{\mathrm{L}}}}{\rho_{\mathrm{e}}\left(\omega_{0}^{2}-V_{\mathrm{Ae}}^{2} k^{2}\right)}-\frac{C_{\mathrm{L} 0} \mathrm{e}^{-\tau_{\mathrm{L}}}}{\rho_{\mathrm{L}}\left(\omega_{0}^{2}-V_{\mathrm{AL}}^{2} k^{2}\right)}=0, \\
& \frac{C_{10} \mathrm{e}^{\tau_{\mathrm{R}}}-C_{20} \mathrm{e}^{-\tau_{\mathrm{R}}}}{\rho_{\mathrm{e}}\left(\omega_{0}^{2}-V_{\mathrm{Ae}}^{2} k^{2}\right)}+\frac{C_{\mathrm{R} 0} \mathrm{e}^{-\tau_{\mathrm{R}}}}{\rho_{\mathrm{R}}\left(\omega_{0}^{2}-V_{\mathrm{AR}}^{2} k^{2}\right)}=0 .
\end{aligned}
$$

This system has a non-trivial solution only when its determinant is zero. This gives the dispersion equation determining $\omega_{0}$ as a function of $k$. The system of Eqs. (66)-(69) is the same as the system of Eqs. (29) in Van Doorsselaere et al. (2008), so that we can use the results obtained by these authors. It follows from their analysis that the solution to the dispersion equation is $\omega_{0}^{2}=\omega_{ \pm}^{2}$, where

$$
\begin{aligned}
& \omega_{ \pm}^{2}=\frac{V_{\mathrm{Ae}}^{2} k^{2}\left(\zeta_{\mathrm{L}}+\zeta_{\mathrm{R}}+2 \pm Q\right)}{\left(\zeta_{\mathrm{L}}+1\right)\left(\zeta_{\mathrm{R}}+1\right)-\left(\zeta_{\mathrm{L}}-1\right)\left(\zeta_{\mathrm{R}}-1\right) E^{2}}, \\
& \zeta_{\mathrm{L}}=\frac{\rho_{\mathrm{L}}}{\rho_{\mathrm{e}}}, \quad \zeta_{\mathrm{R}}=\frac{\rho_{\mathrm{R}}}{\rho_{\mathrm{e}}}, \quad E=\exp \left[-\left(\tau_{\mathrm{L}}+\tau_{\mathrm{R}}\right)\right], \\
& Q=\sqrt{\left(\zeta_{\mathrm{L}}-\zeta_{\mathrm{R}}\right)^{2}+4\left(\zeta_{\mathrm{L}}-1\right)\left(\zeta_{\mathrm{R}}-1\right) E^{2}}
\end{aligned}
$$

Note that, while Luna et al. (2008) found four different eigenfrequencies, we obtained only two, $\omega_{+}$and $\omega_{-}$. As it has been already suggested by Van Doorsselaere et al. (2008), the most probable cause of this difference is that we have neglected the wave dispersion related to the fact that the ratio of the tube radius to its length is small but finite.

Luna et al. (2008) gave an excellent physical explanation why there are high- and low-frequency modes (in their case two high- and two low-frequency modes) of the kink oscillations of two parallel magnetic tubes. Not repeating their explanation we only mention that it is based on the analysis of the plasma motion outside the tubes. In the high-frequency modes this plasma motion provides an additional restoring force thus increasing the oscillation frequency. In the low-frequency modes it reduces the restoring force thus decreasing the oscillation frequency. 


\subsubsection{First-order approximation}

In the first order approximation we collect terms proportional to $C_{11}, C_{21}, C_{\mathrm{L} 1}, C_{\mathrm{R} 1}, \omega_{1}, \ell_{\mathrm{L}}$ or $\ell_{\mathrm{R}}$ in Eqs. (59)-(62). As a result we obtain

$$
\begin{aligned}
C_{11} \mathrm{e}^{-\tau_{\mathrm{L}}} & +C_{21} \mathrm{e}^{\tau_{\mathrm{L}}}-C_{\mathrm{L} 1} \mathrm{e}^{-\tau_{\mathrm{L}}}=\frac{C_{10} \mathrm{e}^{-\tau_{\mathrm{L}}}-C_{20} \mathrm{e}^{\tau_{\mathrm{L}}}}{\rho_{\mathrm{e}}\left(\omega_{0}^{2}-V_{\mathrm{Ae}}^{2} k^{2}\right)} \\
& \times \int_{-\tau_{\mathrm{L}}-\ell_{\mathrm{L}}}^{-\tau_{\mathrm{L}}} \bar{\rho}_{\mathrm{L}}(\tau)\left[\omega_{0}^{2}-k^{2} V_{\mathrm{A}}^{2}(\tau)\right] \mathrm{d} \tau-C_{\mathrm{L} 0} \ell_{\mathrm{L}} \mathrm{e}^{-\tau_{\mathrm{L}}} \\
C_{11} \mathrm{e}^{\tau_{\mathrm{R}}} & +C_{21} \mathrm{e}^{-\tau_{\mathrm{R}}}-C_{\mathrm{R} 1} \mathrm{e}^{-\tau_{\mathrm{R}}}=\frac{C_{10} \mathrm{e}^{\tau_{\mathrm{R}}}-C_{20} \mathrm{e}^{-\tau_{\mathrm{R}}}}{\rho_{\mathrm{e}}\left(\omega_{0}^{2}-V_{\mathrm{Ae}}^{2} k^{2}\right)} \\
& \times \int_{\tau_{\mathrm{R}}}^{\tau_{\mathrm{R}}+\ell_{\mathrm{R}}} \bar{\rho}_{\mathrm{R}}(\tau)\left[\omega_{0}^{2}-k^{2} V_{\mathrm{A}}^{2}(\tau)\right] \mathrm{d} \tau-C_{\mathrm{R} 0} \ell_{\mathrm{R}} \mathrm{e}^{-\tau_{\mathrm{R}}} \\
\left.C_{11} \mathrm{e}^{-\tau_{\mathrm{L}}}-C_{21} \mathrm{e}^{\tau_{\mathrm{L}}}-\frac{C_{\mathrm{L} 1} \mathrm{e}^{-\tau_{\mathrm{L}}}}{\rho_{0}^{2}}-V_{\mathrm{Ae}}^{2} k^{2}\right) & \frac{\mathrm{i} \tau}{\rho_{\mathrm{L}}\left(\omega_{0}^{2}-V_{\mathrm{AL}}^{2} k^{2}\right)}=\left(C_{10} \mathrm{e}^{-\tau_{\mathrm{L}}}+C_{20} \mathrm{e}^{\tau_{\mathrm{L}}}\right) \\
\times & \left(\mathcal{P} \int_{-\tau_{\mathrm{L}}-\ell_{\mathrm{L}}}^{-\tau_{\mathrm{L}}} \frac{\bar{\rho}_{\mathrm{L}}(\tau)\left[\omega_{0}^{2}-k^{2} V_{\mathrm{A}}^{2}(\tau)\right]}{\rho_{\mathrm{AL}}\left|\Delta_{\mathrm{L}}\right|}\right) \\
& +2 \omega_{0} \omega_{1}\left(\frac{C_{10} \mathrm{e}^{-\tau_{\mathrm{L}}}-C_{20} \mathrm{e}^{\tau_{\mathrm{L}}}}{\rho_{\mathrm{e}}\left(\omega_{0}^{2}-V_{\mathrm{Ae}}^{2} k^{2}\right)^{2}}-\frac{C_{\mathrm{L} 0} \mathrm{e}^{-\tau_{\mathrm{L}}}}{\rho_{\mathrm{L}}\left(\omega_{0}^{2}-V_{\mathrm{AL}}^{2} k^{2}\right)^{2}}\right) \\
& -\frac{C_{\mathrm{L} 0} \ell_{\mathrm{L}} \mathrm{e}^{-\tau_{\mathrm{L}}}}{\rho_{\mathrm{L}}\left(\omega_{0}^{2}-V_{\mathrm{AL}}^{2} k^{2}\right)},
\end{aligned}
$$

$$
\begin{aligned}
\frac{C_{11} \mathrm{e}^{\tau_{\mathrm{R}}}}{\rho_{\mathrm{e}}\left(\omega_{0}^{2}-\right.}-C_{21}^{2} \mathrm{e}^{-\tau_{\mathrm{R}}} & +\frac{C_{\mathrm{R} 1} \mathrm{e}^{-\tau_{\mathrm{R}}}}{\rho_{\mathrm{R}}\left(\omega_{0}^{2}-V_{\mathrm{AR}}^{2} k^{2}\right)}=\left(C_{10} \mathrm{e}^{\tau_{\mathrm{R}}}+C_{20} \mathrm{e}^{-\tau_{\mathrm{R}}}\right) \\
& \times\left(\frac{\mathrm{i} \pi}{\rho_{\mathrm{AR}}\left|\Delta_{\mathrm{R}}\right|}-\mathcal{P} \int_{\tau_{\mathrm{R}}}^{\tau_{\mathrm{R}}+\ell_{\mathrm{R}}} \frac{\mathrm{d} \tau}{\bar{\rho}_{\mathrm{R}}(\tau)\left[\omega_{0}^{2}-k^{2} V_{\mathrm{A}}^{2}(\tau)\right]}\right) \\
& +2 \omega_{0} \omega_{1}\left(\frac{C_{10} \mathrm{e}^{\tau_{\mathrm{R}}}-C_{20} \mathrm{e}^{-\tau_{\mathrm{R}}}}{\rho_{\mathrm{e}}\left(\omega_{0}^{2}-V_{\mathrm{Ae}}^{2} k^{2}\right)^{2}}+\frac{C_{\mathrm{R} 0} \mathrm{e}^{-\tau_{\mathrm{R}}}}{\rho_{\mathrm{R}}\left(\omega_{0}^{2}-V_{\mathrm{AR}}^{2} k^{2}\right)^{2}}\right) \\
& +\frac{C_{\mathrm{R} 0} \ell_{\mathrm{R}} \mathrm{e}^{-\tau_{\mathrm{R}}}}{\rho_{\mathrm{R}}\left(\omega_{0}^{2}-V_{\mathrm{AR}}^{2} k^{2}\right)} .
\end{aligned}
$$

$$
\text { recti }
$$
resonant absorption. In the next section we will study the dependence of $\gamma$ on the parameters of the equilibrium.

\section{Investigation of oscillation damping}

In this section we study the dependence of the decrement $\gamma$ on the relative distance between the tube axes, $d / R$, and on the density contract $\zeta$. In what follows we assume that $\bar{\rho}$ is a linear function of $\tau$, so that

$\bar{\rho}=\rho_{\mathrm{e}}\left(1+(\zeta-1) \frac{\tau-\tau_{0}}{\ell}\right)$.

Recall that the Alfvén resonant position $\tau_{\mathrm{A}}$ is determined by the equation $k V_{\mathrm{A}}\left(\tau_{\mathrm{A}}\right)=\omega_{ \pm}$. It follows from this equation that

$\rho_{\mathrm{A}}=\frac{B^{2} k^{2}}{\mu_{0} \omega_{ \pm}^{2}}=\frac{\rho_{\mathrm{e}} V_{\mathrm{Ae}}^{2} k^{2}}{\omega_{ \pm}^{2}}$.

Using this result we obtain

Equations (73)-(76) constitute the system of linear inhomogeneous algebraic equations. The homogeneous counterpart of this system coincides with the system of Eqs. (66)-(69), so that it has a non-trivial solution. This means that its determinant is zero, so that the system of inhomogeneous Eqs. (73)-(76) is solvable only if its right-hand side satisfies the compatibility condition.

At this point calculations are getting increasingly complicated. For the sake of simplicity we restrict further analysis to the case of two identical tubes. In accordance with this we drop the subscripts " $L$ " and " $R$ " and introduce the notation

$\tau_{0}=\tau_{\mathrm{L}}=\tau_{\mathrm{R}}, \quad \bar{\rho}_{\mathrm{L}}(-\tau)=\bar{\rho}_{\mathrm{R}}(\tau)=\bar{\rho}(\tau)$.

For this particular case

$\omega_{ \pm}^{2}=\frac{2 k^{2} V_{\mathrm{Ae}}^{2}}{(\zeta+1) \mp(\zeta-1) E}, \quad \frac{C_{0 \mathrm{R}}}{C_{0 \mathrm{~L}}}= \pm 1$.

Using Eqs. (68), (69), (77) and (78) we obtain that the compatibility condition for the system of Eqs. (73)-(76) reads

$$
\begin{aligned}
2 \omega_{ \pm} \omega_{1} & +\rho_{\mathrm{e}} V_{\mathrm{Ae}}^{4} k^{4} \frac{(\zeta-1)^{2}\left(1-\mathrm{e}^{-4 \tau_{0}}\right)\left(1 \pm \mathrm{e}^{-2 \tau_{0}}\right)}{\left[(\zeta+1) \mp \mathrm{e}^{-2 \tau_{0}}(\zeta-1)\right]^{3}} \\
& \times\left(\frac{\mathrm{i} \pi}{\rho_{\mathrm{A}}|\Delta|}-\mathcal{P} \int_{\tau_{0}}^{\tau_{0}+\ell} \frac{\mathrm{d} \tau}{\bar{\rho}\left(\omega_{ \pm}^{2}-k^{2} V_{\mathrm{Ae}}^{4}\right)}\right)=0 .
\end{aligned}
$$

$$
\begin{aligned}
\Delta=-\left.k^{2} \frac{\mathrm{d} V_{\mathrm{A}}^{2}}{\mathrm{~d} \tau}\right|_{\tau=\tau_{\mathrm{A}}} & =\left.\frac{B^{2} k^{2}}{\mu_{0} \bar{\rho}^{2}} \frac{\mathrm{d} \bar{\rho}}{\mathrm{d} \tau}\right|_{\tau=\tau_{\mathrm{A}}}=\frac{\rho_{\mathrm{e}} B^{2} k^{2}(\zeta-1)}{\mu_{0} \rho_{\mathrm{A}}^{2} \ell} \\
& =\frac{\rho_{\mathrm{e}} \mu_{0} \omega_{ \pm}^{4}(\zeta-1)}{\ell B^{2} k^{2}}=\frac{\omega_{ \pm}^{4}(\zeta-1)}{\ell k^{2} V_{\mathrm{Ae}}^{2}} .
\end{aligned}
$$

Using Eqs. (78), (84) and (85) we obtain from Eq. (82) that

$\gamma_{ \pm}=\frac{\pi \ell \omega_{ \pm}(\zeta-1)\left(1-\mathrm{e}^{-4 \tau_{0}}\right)\left(1 \pm \mathrm{e}^{-2 \tau_{0}}\right)}{8\left[(\zeta+1) \mp \mathrm{e}^{-2 \tau_{0}}(\zeta-1)\right]}$

It was found in studies of resonant damping of kink oscillations of single magnetic loops that the decrement is proportional to the ratio of the inhomogeneous annulus thickness to the loop radius (see, e.g., Ruderman \& Roberts 2002; Goossens et al. 2002; Goossens et al. 2006; Ruderman \& Erdélyi 2009). To compare the damping rate of kink oscillations of two parallel loops with that of a single loop it would be convenient to write the expression for $\gamma_{ \pm}$in the same form. We see from Eq. (86) that $\gamma_{ \pm}$is proportional to $\ell$. However $\ell$ is equal to the ratio of the annulus thickness to the loop radius only when the distance between the loop axes is much larger than the loop radius. It would be desirable to express $\ell$ in terms of this ratio in the general case. The difficulty is that, in general, the annulus thickness is not constant. Rather it is a function of $\sigma$. To overcome this difficulty we introduce the mean thickness of the annulus. The equations of the external and internal boundaries of the annulus in Cartesian coordinates are given by Eq. (1) with $\tau$ substituted by $\tau_{0}$ and $\tau_{0}+\ell$ 
respectively. Then, taking into account that $\ell \ll \tau_{0}$, we obtain that the annulus thickness at fixed $\sigma$ is given by the approximate formula

$\delta(\sigma)=\ell\left[\left(\frac{\partial x}{\partial \tau}\right)^{2}+\left(\frac{\partial y}{\partial \tau}\right)^{2}\right]^{1 / 2}=\frac{a \ell}{\cosh \tau_{0}-\cos \sigma}$.

The mean annulus thickness is given by

$\bar{\delta}=\frac{1}{2 \pi R} \int_{0}^{2 \pi} \delta(\sigma) \mathrm{d} s(\sigma)$

The square of the elementary length along the annulus boundary is given by

$$
\begin{aligned}
\mathrm{d} s^{2}=\mathrm{d} x^{2}+\mathrm{d} y^{2} & =\left[\left(\frac{\partial x}{\partial \sigma}\right)^{2}+\left(\frac{\partial y}{\partial \sigma}\right)^{2}\right] \mathrm{d} \sigma^{2} \\
& =\frac{a^{2} \mathrm{~d} \sigma^{2}}{\left(\cosh \tau_{0}-\cos \sigma\right)^{2}} .
\end{aligned}
$$

In particular, it follows from this result that the length of the annulus boundary is given by

$2 \pi R=\int_{0}^{2 \pi} \mathrm{d} s(\sigma)=a \int_{0}^{2 \pi} \frac{\mathrm{d} \sigma}{\cosh \tau_{0}-\cos \sigma}$.

Using Eqs. (87) and (89) we obtain from Eq. (88) that

$\bar{\delta}=\frac{a^{2} \ell}{2 \pi R} \int_{0}^{2 \pi} \frac{\mathrm{d} \sigma}{\left(\cosh \tau_{0}-\cos \sigma\right)^{2}}$

Substituting in Eq. (90) $R=a / \sinh \tau_{0}$ and differentiating the obtained identity with respect to $\tau_{0}$ we obtain

$$
\int_{0}^{2 \pi} \frac{\mathrm{d} \sigma}{\left(\cosh \tau_{0}-\cos \sigma\right)^{2}}=\frac{2 \pi \cosh \tau_{0}}{\sinh ^{3} \tau_{0}} .
$$

Substituting this result in Eq. (91) yields

$\bar{\delta}=\frac{2 \pi a^{2} \ell \cosh \tau_{0}}{2 \pi R \sinh ^{3} \tau_{0}}=\frac{a \ell \cosh \tau_{0}}{\sinh ^{2} \tau_{0}}=\ell R \operatorname{coth} \tau_{0}$.

Using this result we rewrite Eq. (86) as

$\gamma_{ \pm}=\frac{\pi \bar{\delta} \omega_{ \pm}(\zeta-1)\left(1 \pm \mathrm{e}^{-2 \tau_{0}}\right)\left(1-\mathrm{e}^{-2 \tau_{0}}\right)^{2}}{8 R\left[(\zeta+1) \mp \mathrm{e}^{-2 \tau_{0}}(\zeta-1)\right]}$

This formula is quite convenient for comparison with the damping rate obtained for a single loop. First of all we note that the damping rate is proportional to the annulus thickness. The same result was obtained for a single magnetic tube in the thin boundary layer approximation (e.g. Ruderman \& Roberts 2002; Goossens et al. 2006). When $\tau_{0} \rightarrow \infty$ we obtain $d / R \rightarrow \infty$. In this case the loops oscillate independently, and the annulus thickness is constant, $\delta(\sigma)=\bar{\delta}$. When $\tau_{0} \rightarrow \infty$ we obtain $\omega_{ \pm}^{2}=2 V_{\mathrm{Ae}}^{2} k^{2} /(\zeta-1) \equiv \omega_{k}^{2}$ and

$\gamma_{ \pm}=\frac{\pi \bar{\delta} \omega_{k}(\zeta-1)}{8 R(\zeta+1)}$

This result is in a complete agreement with that obtained for a single loop (see, e.g., Goossens et al. 2002; Ruderman \& Erdélyi 2009). The dependence of $\gamma_{ \pm} R / \bar{\delta} \omega_{ \pm}$on $d / R$ and $\zeta$ is shown in in Figs. 2 and 3 respectively. We can clearly see that the the decrement is an increasing function of both the separation distance and the density contrast. We can also see both from Eq. (95) and Figs. 2 and 3 that the faster mode damps more efficiently than the slower mode.

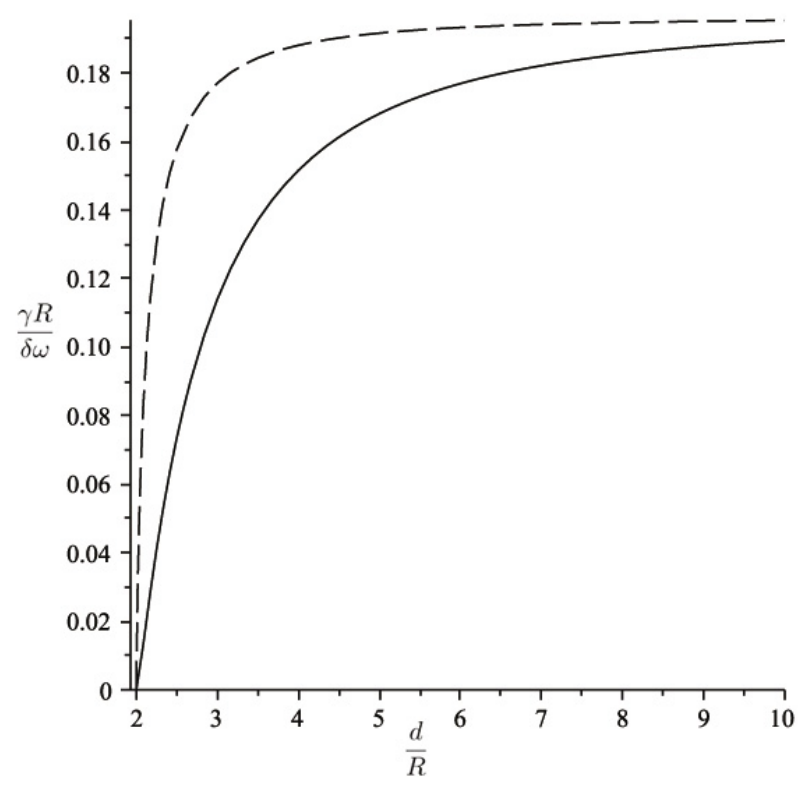

Fig. 2. The dependence of the decrement $\gamma$ on the distance between the loop axes $d$ for $\zeta=3$. The solid and dashed curves correspond to the slower $\left(\omega=\omega_{-}\right)$and faster $\left(\omega=\omega_{+}\right)$kink oscillations.

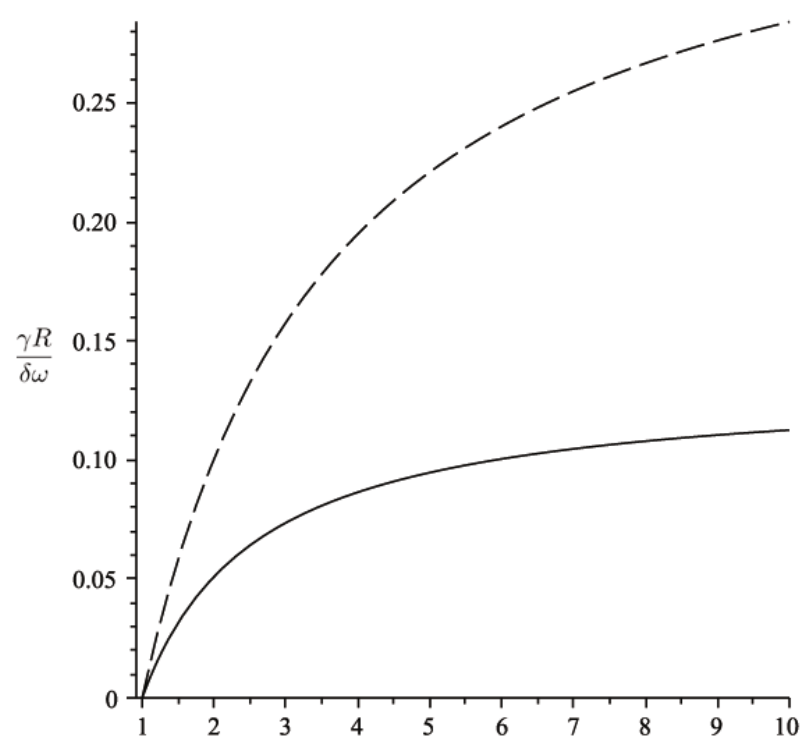

Fig. 3. The dependence of the decrement $\gamma$ on the density contrast $\zeta$ for $d / R=2.5$. The solid and dashed curves correspond to the slower $\left(\omega=\omega_{-}\right)$and faster $\left(\omega=\omega_{+}\right)$kink oscillations.

\section{Summary and conclusions}

In this paper we have studied the damping of kink oscillations of the system of two parallel straight magnetic tubes. The density has been assumed to be constant outside the tubes and in the core regions of the tubes, while it was varying in the radial direction in thin annuluses near the tube boundaries. As a result there are resonant positions in the annuluses. There is one resonant position in each annulus in a system of standard type both for modes with lower and higher frequencies, while in a system of anomalous type there are still two resonant positions for modes with higher frequencies, but only one resonant position in the annulus in a denser tube for modes with lower frequencies.

The assumption that the annuluses are thin enabled us to use the regular perturbation method to study the damping of kink 
oscillations due to resonant absorption. In the zero-order approximation we reproduced the results of studying kink oscillations of two homogeneous tubes obtained by Van Doorsselaere et al. (2008). In the first-order approximation we calculated the damping rate due to resonant absorption. At this stage calculations are getting increasingly complicated, so that we restricted our analysis to the case of identical magnetic tubes.

The main results concerning the damping of oscillations can be summarized as follows. We can see from Fig. 2 that, for $\zeta=3$, the decrements of both slower and faster kink modes are monotonically increasing functions of $d / R$. Taking into account that $d / R=\cosh \tau_{0}$, i.e. $d / R$ is a monotonically increasing function of $\tau_{0}$, it is straightforward to show that the same result is valid for any value $\zeta>1$. Hence, the interaction between the tubes reduces the efficiency of resonant damping. Formally, $\gamma_{ \pm} \rightarrow 0$ as $d / R \rightarrow 2$. However, the assumption that the annuluses are thin implies that not only $\bar{\delta} \ll R$, but also $\bar{\delta}$ is much smaller than the distance between the tube boundaries. This second restriction is equivalent to $d-2 R \gg \bar{\delta}$.

Another important conclusion concerning the damping rate is that $\gamma_{-} / \omega_{-}<\gamma_{+} / \omega_{+}$, i.e. the damping time of slower oscillations is larger than that of faster oscillations. For example, for $\zeta=3$ and $d / R=2.5$, the damping time of slower oscillations is more than twice larger than that of faster oscillations. At present we cannot provide a physical explanation to this result. Finally, similar to the case of a single tube, the damping time is an increasing function of the density contrast $\zeta$.

It is interesting to compare our results with those obtained by Arregui et al. (2007). In their study of resonantly damped kink oscillations of the system of two parallel slabs these authors found that the properties of kink oscillations of this system and the damping time are practically the same as of a single slab with the equivalent parameters. In this sense the properties of kink oscillations of a system of two tubes are quite different from those of two-slab system. While Arregui et al. (2007) found only one fundamental eigenfrequency of kink oscillations, we found two of them and, as we know from the study by Luna et al. (2008), actually there are four eigenfrequencies if the wave dispersion is taken into account. We also found that the damping rate due to resonant absorption is sufficiently reduced by the tube interaction. This difference between our results and those by Arregui et al. (2007) is not very surprising. As we have already noted, the properties of kink oscillations of single slabs and tubes are quite different. It is then natural to expect a substantial difference in properties of kink oscillations of two-tube and two-slab systems.

One final note is that the result that the damping rate decreases when the tube separation decreases should be treated with caution. The point is that, when studying the damping rate, we fixed the average thickness of the annuluses where the density decreases from its value inside the tubes to its external value.
However the shape of annuluses changes when the tube separation decreases. Their thickness is getting larger in the outer parts of the tubes and smaller in the inner part. It is possible that the damping rate decrease is related to this change of annulus shape. To clarify this point one needs to study the effect of annulus shape on the damping rate. In this respect we note that this problem has not been addressed yet even in the case of a single magnetic tube. So it is an interesting problem for future studies.

Acknowledgements. M.S.R. acknowledges the support by an STFC grant. D.R. acknowledges the support by an STFC postgraduate fellowship.

\section{References}

Andries, J., Goossens, M., Hollweg, J. V., Arregui, I., \& Van Doorsselaere, T. 2005, A\&A, 430, 1109

Arregui, I., Terradas, J., Oliver, R., \& Ballester, J. L. 2007, A\&A, 466, 1145

Arregui, I., Terradas, J., Oliver, R., \& Ballester, J. L. 2008, ApJ, 674, 1179

Aschwanden, M. J. 2005, ApJ, 634, L193

Aschwanden, M. J., \& Nightingale, R. W. 2005, ApJ, 633, 499

Aschwanden, M. J., Fletcher, L., Schrijver, C. J., \& Alexander, D. 1999, ApJ, 520,880

Aschwanden, M. J., De Pontieu, B., Schrijver, C. J., \& Tilte, A. M. 2002, Sol. Phys., 206, 99

Braginskii, S. I., 1965, in Review of Plasma Physics, ed. A. V. Leontovich (New York: Consultants Bureau), 1, 205

Dymova, M. V., \& Ruderman, M. S. 2006, A\&A, 457, 1059

Erdélyi, R., \& Goossens, M. 1995, A\&A 294, 575

Goossens, M., \& Ruderman, M. S. 1995, Phys. Scripta, T60, 171

Goossens, M., Ruderman, M. S., \& Hollweg, J. V. 1995, Sol. Phys., 157, 75

Goossens, M., Andries, J., \& Aschwanden, M. J. 2002, A\&A, 394, L39

Goossens, M., Andries, J., \& Arregui, I. 2006, Phil. Trans. R. Soc. A, 364, 433

Hollweg, J. V. 1987, ApJ, 312, 880

Korn, G., \& Korn, T. 1961, Mathematical handbook for scientists and engineers (New York: McGraw-Hill)

Luna, M., Terradas, J., Oliver, R., \& Ballester, J. L. 2008, ApJ, 676, 717

Luna, M., Terradas, J., Oliver, R., \& Ballester, J. L. 2009, ApJ, 692, 1582

Luna, M., Terradas, J., Oliver, R., \& Ballester, J. L. 2010, ApJ, 716, 1371

Martens, P. C. H., Cirtain, J. W., \& Schmelz, J. T. 2002, ApJ, 577, L115

Morton, R. J, \& Erdelyi, R. 2009, ApJ, 707, 750

Nakariakov, V. M., Ofman, L., DeLuca, E. E., Roberts, B., \& Davila, J. M. 1999 Science, 285,862

Ofman, L. 2005, Adv. Space Res., 36, 1572

Ofman, L. 2009, ApJ, 694, 502

Ofman, L., \& Aschwanden, M. J. 2002, ApJ, 576, L153

Ofman, L., Davila, J. M., \& Steinolfson, R. S. 1994, ApJ, 421, 360

Robertson, D., Ruderman, M. S., \& Taroyan Y. 2010, A\&A, 515, A33

Ruderman, M. S., \& Erdélyi, R. 2009, Space. Sci. Rev., 149, 199

Ruderman, M. S., \& Roberts, R. 2002, ApJ, 577, 475

Ruderman, M. S., Tirry, W., \& Goossens, M. 1995, J. Plasma Phys., 54, 129

Schmelz, J. T., Cirtain, J. W., Beene, J. E., et al. 2003, Adv. Space Res., 32, 1109

Schmelz, J. T., Nasraoui, K., Richardson, V. L., et al. 2005, ApJ, 627, L81

Schrijver, C. J., Aschwanden, M. J., \& Tilte, A. M. 2002, Sol. Phys., 206, 69

Terradas, J., Arregui, I., Oliver, R., et al. 2008, ApJ, 679, 1611

Tirry, W. J., \& Goossens, M. 1996, ApJ, 471, 501

Van Doorsselaere, T., Ruderman, M. S., \& Robertson, D. 2008, A\&A, 485, 849

Verwichte, E., Nakariakov, V., Ofman, L., \& DeLuca, E. E. 2004, Sol. Phys. 223,77 